\title{
Impact of Prior Cancer History on the Clinical Outcomes in Advanced Breast Cancer: A Propensity Score-Adjusted, Population-Based Study
}

\author{
Caijin Lin, MD \\ Jiayi Wu, MD \\ Shuning Ding, MD \\ Chihwan Goh, MD \\ Lisa Andriani, MD \\ Kunwei Shen, MD, PhD \\ Li Zhu, MD, PhD
}

Comprehensive Breast Health Center, Ruijin Hospital, Shanghai Jiao Tong University

School of Medicine, Shanghai, China

\begin{abstract}
Purpose
Despite the rapid growing of cancer survivors, prior cancer history is a commonly adopted exclusion criterion. Whether prior cancer will impact the survival of patients with advanced breast cancer $(A B C)$ remains uncertain.
\end{abstract}

\section{Materials and Methods}

Patients with ABC diagnosed between 2004 and 2010 were identified using Surveillance, Epidemiology, and End Results (SEER) database. Timing, stage, and type were used to characterize prior cancer. Multivariable analyses using propensity score-adjusted Cox regression and competing risk regression were conducted to evaluate the prognostic effect of prior cancer on overall survival (OS) and breast cancer-specific survival (BCSS).

\section{Results}

A total of $14,176 \mathrm{ABC}$ patients were identified, of whom $10.5 \%$ carried a prior cancer history. The most common type of prior cancer was female genital cancer (32.4\%); more than half (51.7\%) were diagnosed at localized stage; most were diagnosed more than 5 years $(42.9 \%)$ or less than 1 year (28.3\%) prior to the index cancer. In multivariate analyses, patients with prior cancer presented a slightly worse OS (hazard ratio, 1.18; 95\% confidence interval [CI], 1.07 to 1.30; $\mathrm{p}=0.001$ ) but a better BCSS (subdistribution hazard ratio, 0.64; 95\% Cl, 0.56 to $0.74 ; p<0.001$ ). In subset analyses, no survival detriment was observed in patients with prior malignancy from head and neck or endocrine system, at in situ or localized stage, or diagnosed more than 4 years.

\section{Conclusion}

Prior cancer provides an inferior OS but a superior BCSS for patients with ABC. It does not affect the survival adversely in some subgroups and these patients should not be excluded from clinical trials.
Tel: 86-13701691557

Fax: 86-21-64156886

E-mail: zhuli8@yeah.net

Received April 19, 2019

Accepted November 14, 2019

Published Online November 18, 2019

${ }^{*}$ Caijin Lin and Jiayi Wu contributed equally to this work.
Key words

Breast neoplasms, Prior cancer, Prognosis, Clinical trial, Competing risk

\section{Introduction}

Breast cancer is the most common malignancy for women in the United States [1]. The incidence rate of breast cancer is reported to increase by $0.4 \%$ per year, while the death rate declines annually by $1.6 \%$ [2]. These data suggest a rapid growing of cancer survivors, leading to the prevalence of multiple primary cancers [3]. As is reported, about $4 \%$ to $14 \%$ of patients diagnosed with breast cancer carry a history of prior cancer $[4,5]$.

The National Comprehensive Cancer Network believes that being in clinical trials is the best management for patients with cancer [6]. However, fewer than $5 \%$ of cancer patients can participate in clinical trials due to excessive or overly restrictive exclusion criteria [7,8], one of which is a history of prior malignancy. For example, more than $80 \%$ of lung cancer trials sponsored by the Eastern Cooperative Oncology Group (ECOG) exclude cancer survivors [9]. Besides, there are many clinical trials of advanced breast cancer $(\mathrm{ABC})$ 
excluding patients with prior cancer within the timeframe of 3 years and some exclude patients with prior cancer diagnosed at any time. The practice is largely attributed to the long-held belief that a history of prior cancer may influence the trial conduct and outcomes. While the Food and Drug Administration introduced a draft guidance recently suggesting that patients with a history of prior malignancy should generally be enrolled in clinical trials [10]. Similarly, Laccetti et al. [11] found that prior cancer did not adversely affect clinical outcomes for lung cancer $[9,12]$. However, the prognostic impact of a prior cancer history varies among different cancer types and stage in question [13], and the reasonableness of this criterion has not been assessed in patients with $\mathrm{ABC}$.

Therefore, we conducted the study to determine the impact of prior cancers on clinical outcomes among patients with $\mathrm{ABC}$ using the Surveillance, Epidemiology, and End Results (SEER) database.

\section{Materials and Methods}

\section{Database and case selection}

The population-based data were obtained from the SEER Program of the National Cancer Institute, which covers approximately $28 \%$ of the U.S. population [14]. We selected patients with ABC diagnosed between January 2004 and December 2010 using SEER ${ }^{*}$ Stat software (ver. 8.3.5, https: // seer.cancer.gov/seerstat) to ensure a 5-year follow-up at least. Breast cancer was identified by site codes (C50.0-C50.6, C50.8, and C50.9) of the International Classification of Diseases for Oncology, 3rd edition (ICD-O-3). Patients were identified if they had stage IV disease according to the 6th edition of the American Joint Committee on Cancer (AJCC) Cancer Staging Manual. We excluded patients younger than 18 years old at diagnosis, those with incomplete follow-up information including diagnosis dates and survival data, or patients diagnosed at autopsy or via death certificate. Besides, we further excluded patients with a diagnosis of prior breast cancer. Actually, it is challenging to distinguish the primary cancer from recurrent disease when there is a same-site second cancer even in the clinical practice [11]. Detailed inclusion and exclusion criteria were included in the online supplementary materials.

A history of primary cancer was derived from the SEER sequence number, which described the sequence of all reportable malignancy over the lifetime of a patient. The sequence number of " 00 " suggested that the patient had only one primary cancer in the lifetime. For women with multiple primaries, the sequence number of " 01 " indicated the first cancer and " 02 " indicated the second one, and so forth. In another word, patients without prior cancer were those whose sequence number were " 00 " or " 01. "

\section{Variables}

Demographic and clinicopathological data were extracted from the SEER database, including age, race, marital status, histology, pathologic grade, status of estrogen receptor (ER) and progesterone receptor (PR), SEER stage, surgery of the primary tumor and chemotherapy. All eligible patients in our study were female. Race/ethnicity was categorized as white, black, Hispanic, and others according to SEER coding manual 2016. Marital status was classified as married, single, separated/widowed/divorced (sep/wid/div) and unknown. According to the SEER terminology, the extent of the disease was described as localized, regional, distant and unstaged. The stage and type of the most recent prior cancer were also recorded in detail. The timing, namely the interval between the diagnosis of prior cancer and index breast cancer, was calculated based on the diagnosis date.

\section{Statistical analysis}

Pearson chi-square tests were used to analyze categorical variables. The primary outcomes were overall survival (OS) and breast cancer-specific survival (BCSS). Kaplan-Meier method and Gray's test were used to estimate OS and BCSS of no prior cancer vs any prior cancer diagnosis, respectively, before and after propensity score matching (PSM) [15]. Survival curves stratified by timing of prior cancer were also constructed based on the Kaplan-Meier method. Both unadjusted and propensity score-adjusted Cox proportional hazards models were built to identify whether the prior cancer history was an independent prognostic factor for OS. To test the impact on BCSS of the prior cancer diagnosis, Fine and Gray's competing risks regression was also performed before and after PSM [16]. Since the proportional hazards assumption did not hold based on the Schoenfeld residuals, we included the interactions of covariates with functions of time in the multivariable models $[17,18]$. After the introduction of time-dependent covariates, only slight shift of hazard ratios (HR) and the corresponding confidence interval (CI) were observed. A possible explanation for the disproportion might be that it was easy to detect the small departures in a large dataset [11]. A two-sided p-value of less than 0.05 indicated statistical significance. All these procedures were conducted in R software (ver. 3.5.1, http:// www.r-project.org). 
Table 1. Baseline characteristics of patients diagnosed with advanced breast cancer before propensity score matching

\begin{tabular}{|c|c|c|c|c|}
\hline Characteristic & Total & Prior malignancy & No prior malignancy & p-value \\
\hline \multicolumn{5}{|l|}{ Age (yr) } \\
\hline$<40$ & 969 & $5(1.1)$ & $964(7.1)$ & $<0.001$ \\
\hline $40-64$ & 7,493 & $177(38.0)$ & $7,316(54.2)$ & \\
\hline$>64$ & 5,492 & $284(60.9)$ & $5,208(38.6)$ & \\
\hline \multicolumn{5}{|l|}{ Race } \\
\hline White & 9,269 & $348(74.7)$ & $8,921(66.1)$ & 0.001 \\
\hline Black & 2,300 & $55(11.8)$ & 2,245 (16.6) & \\
\hline Hispanic & 1,395 & $33(7.1)$ & $1,362(10.1)$ & \\
\hline Other & 990 & $30(6.4)$ & $960(7.1)$ & \\
\hline \multicolumn{5}{|l|}{ Marital status } \\
\hline Married & 6,041 & $189(40.6)$ & $5,852(43.4)$ & $<0.001$ \\
\hline Single & 2,791 & $56(12.0)$ & $2,735(20.3)$ & \\
\hline Sep/wid/div & 4,517 & $201(43.1)$ & $4,316(32.0)$ & \\
\hline Unknown & 605 & $20(4.3)$ & $585(4.3)$ & \\
\hline \multicolumn{5}{|l|}{ Histology } \\
\hline IDC & 10,561 & $356(76.4)$ & $10,205(75.7)$ & 0.001 \\
\hline ILC & 1,657 & $73(15.7)$ & $1,584(11.7)$ & \\
\hline Other & 1,699 & $37(7.9)$ & $1,699(12.6)$ & \\
\hline \multicolumn{5}{|l|}{ Grade } \\
\hline G1 & 841 & $32(6.9)$ & $809(6.0)$ & 0.840 \\
\hline G2 & 4,643 & $158(33.9)$ & $4,485(33.3)$ & \\
\hline G3/G4 & 6,247 & $202(43.3)$ & $6,045(44.8)$ & \\
\hline Unknown & 2,223 & $74(15.9)$ & 2,149 (15.9) & \\
\hline \multicolumn{5}{|l|}{ ER } \\
\hline Positive & 9,245 & $284(60.9)$ & $8,961(66.4)$ & 0.001 \\
\hline Negative & 3,617 & $126(27.0)$ & $3,491(25.9)$ & \\
\hline Unknown & 1,092 & $56(12.0)$ & $1,036(7.7)$ & \\
\hline \multicolumn{5}{|l|}{ PR } \\
\hline Positive & 7,228 & $214(45.9)$ & $7,014(52.0)$ & 0.001 \\
\hline Negative & 5,540 & $193(41.4)$ & $5,347(39.6)$ & \\
\hline Unknown & 1,186 & $59(12.7)$ & $1,127(8.4)$ & \\
\hline \multicolumn{5}{|l|}{ Treatment } \\
\hline No & 4,016 & $177(38.0)$ & $3,839(28.5)$ & $<0.001$ \\
\hline Surg only & 2,192 & $95(20.4)$ & 2,097 (15.5) & \\
\hline Chemo only & 3,822 & $99(21.2)$ & $3,723(27.6)$ & \\
\hline Surg and Chemo & 3,924 & $95(20.4)$ & $3,829(28.4)$ & \\
\hline \multicolumn{5}{|l|}{ Cause of death ${ }^{\text {a) }}$} \\
\hline Alive & 2,287 & $46(9.9)$ & 2,241 (16.6) & $<0.001$ \\
\hline Breast cancer specific & 10,040 & $279(59.9)$ & $9,761(72.4)$ & \\
\hline All other sites & 1,627 & $141(30.3)$ & $1,486(11.0)$ & \\
\hline Total & 13,954 & 466 & 13,488 & \\
\hline
\end{tabular}

Values are presented as number (\%). Sep/wid/ div, separated / widowed/ divorced; IDC, infiltrating ductal carcinoma; ILC, infiltrating lobular carcinoma; ER, estrogen receptor; PR, progesterone receptor; Surg, surgery; Chemo, chemotherapy. a)Dependent variable, no necessary to calculate the standardized difference.

\section{PSM analysis}

To minimize the baseline confounders, a one-to-one PSM between women with or without a history of prior cancer was carried out using the nearest-neighbor matching method with a caliper distance of 0.2 [19]. Variables entered into the PSM algorithm included age, race, marital status, histology, pathologic grade, status of ER and PR, surgery of primary 
tumor and chemotherapy. After matching, standardized differences were calculated and a value of less than 0.2 denoted meaningful balance [20]. Besides, the propensity-score adjusted p-value was provided as well. The PSM procedure and the calculation of standardized differences were performed using R packages of MatchIt (ver. 3.0.2), optmatch (ver. 0.9-10), and stddiff (ver. 2.0).

\section{Ethical statement}

All analyses of human data conducted in this study were approved by the independent Ethical Committee/Institutional Review Board of Ruijin Hospital and in accordance with the 1964 Helsinki declaration and its later amendments or comparable ethical standards. Informed consent was exempted because of the retrospective nature of this study.

\section{Results}

In all, 328,092 patients with breast cancer and 14,176 patients with advanced disease were identified, of whom $32,798(10.0 \%)$ and $1,483(10.5 \%)$ carried a history of prior cancer, respectively. After excluding patients with a prior breast cancer diagnosis, a total of 12,126 patients (3.7\%) with breast cancer and 466 patients (3.3\%) with advanced disease were used for further analysis. We could see that prior cancer was more common among older ( $>64$ years old), white and separated/widowed/divorced women. After adjusted for propensity scores, all variables were well-balanced between women with or without prior cancer. An overview of detailed baseline characteristics before and after PSM were provided in Table 1 and S1 Table.

Type, stage, and timing of the most recent prior malignancy were displayed using treemaps in Fig. 1. For women with advanced disease, the most common type included female genital (32.4\%), gastrointestinal (23.4\%), and respiratory $(12.0 \%)$ cancers. Most prior cancers were diagnosed at localized $(51.7 \%)$ or regional $(26.0 \%)$ stage. The median interval between the diagnosis of prior cancer and the index cancer was 50 months. Most were diagnosed within one year $(28.3 \%)$, more than 10 years $(21.9 \%)$ or 5 to 10 years $(21.0 \%)$ prior to the prior cancer diagnosis in patients with stage IV breast cancer. Similar distribution patterns were observed in patients with breast cancer (S2 Fig.).

Overall, the Kaplan-Meier plots showed significantly worse prognosis $(p<0.001)$ for patients with a history of prior cancer after adjusted for propensity scores, presenting an adverse effect of prior cancer on OS. However, a prior cancer diagnosis was found to be a favorable prognostic factor
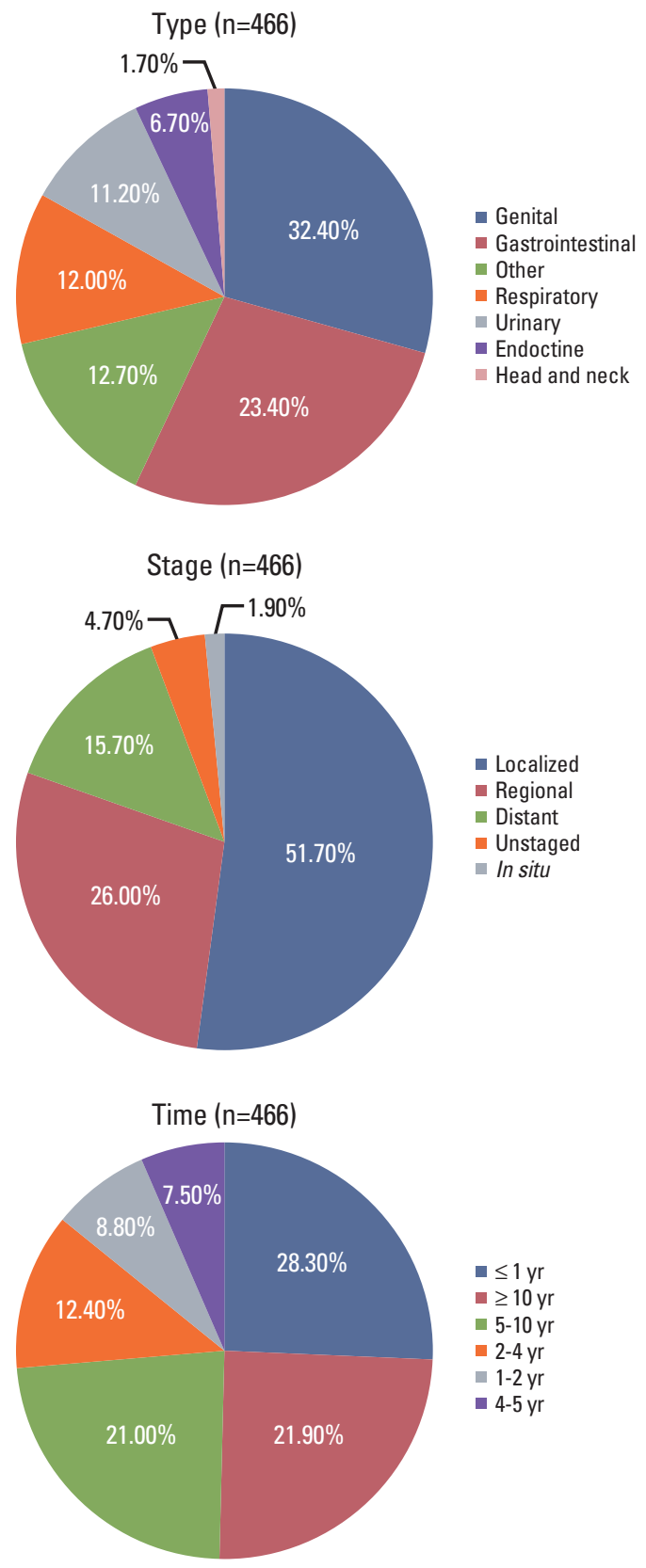

Fig. 1. Type, stage, and time distribution of the most recent prior malignancy for patients with advanced breast cancer.

for BCSS based on the Gray's test (pBCSS $<0.001$ ) for some uncovered reasons (Fig. 2). Both the unfavorable impact on OS $(p<0.001)$ and the favorable impact on BCSS (pBCSS $=0.005$ ) were reaffirmed in the unadjusted survival curves (S3 Fig.).

Fig. 3 demonstrated the impact of timing of prior cancer diagnosis on OS and we found that patients who had a prior 
A
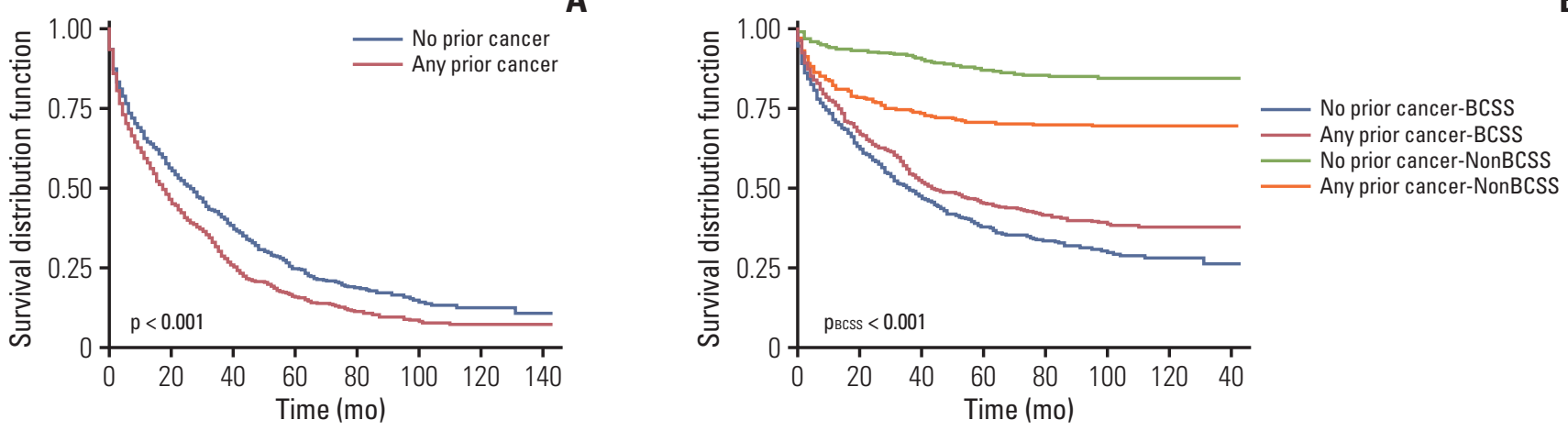

Fig. 2. Overall (A) and breast cancer-specific (B) survival (BCSS) for patients with or without prior cancer diagnosis after propensity score matching. Two-sided p-values were estimated and compared based on the log-rank test (A) and Gray's test (B).

cancer diagnosis with a disease-free interval of 4 years or longer showed a similar survival to those without prior cancer (HR, 1.16; 95\% CI, 0.98 to 1.37; $\mathrm{p}=0.074$ ). Besides, we found that prior cancers from endocrine system (HR, 0.89; $95 \% \mathrm{CI}, 0.56$ to $1.42 ; \mathrm{p}=0.630$ ) or head and neck (HR, 1.24; $95 \% \mathrm{CI}, 0.54$ to $2.83 ; \mathrm{p}=0.618$ ) and prior cancers at in situ (HR, $1.02 ; 95 \% \mathrm{CI}, 0.48$ to $2.18 ; \mathrm{p}=0.962$ ) or localized (HR, $1.15 ; 95 \%$ $\mathrm{CI}, 0.97$ to $1.36 ; \mathrm{p}=0.083$ ) stage might not influence the prognosis significantly. Patients with different timing, stage or type of prior cancer diagnosis presented non-inferior or even slightly superior BCSS to those without prior cancer. Detailed data were presented in Table 2.

After adjusted for age, race, marital status, grade, status of ER and PR, and the treatment modality, a history of prior cancer were found to be an independent prognostic factor for overall (HR, $1.23 ; 95 \% \mathrm{CI}, 1.07$ to $1.41 ; \mathrm{p}=0.004)$ and breast cancer-specific (subdistribution hazard ratio [SHR], 0.75; $95 \%$ CI, 0.63 to $0.88 ; \mathrm{p}<0.001$ ) survival with the opposite direction in the propensity score-adjusted models (Table 3 ). The association remained significant both in the Cox model (HR, 1.18; 95\% CI, 1.07 to 1.30; $\mathrm{p}=0.001$ ) and the competing risks model before PSM (SHR, 0.64; 95\% CI, 0.56 to $0.74 ; \mathrm{p}<0.001$ ) (S4 Table).

\section{Discussion}

This study focused on the impact of a prior cancer diagnosis on clinical outcomes among patients with stage IV breast cancer. First, approximately $10 \%$ of patients were diagnosed with a history of prior cancer and 3\% had a history of different-site prior malignancy. Second, more than half prior can- cers were diagnosed at in situ or localized stage. Furthermore, a prior cancer history was observed to impact overall and cancer-specific survival in the opposite direction. While no survival detriment was observed in patients with prior malignancies at in situ or localized stage or those diagnosed outside the timeframe of 4 years.

Over the past decades, survival for stage IV breast cancer has been improving steadily and about one-third of women with advanced disease live more than 5 years [2,21]. Consistent with the findings reported herein, approximately $7 \%$ to $14 \%$ of patients with breast cancer were reported to have a history of prior breast cancer and about $4 \%$ to $8 \%$ of patients had a prior cancer on different sites in a previous study [4]. Besides, patients older than 64 years old were most commonly diagnosed with a history of prior cancer in the present study. Similar findings were also reported by Murphy et al. [4] and Bluethmann et al. [22]. The rapid growing of cancer survivors urges us to revisit the longstanding practice of excluding a majority of otherwise eligible patients with a history of prior cancer from clinical trials, especially the older patients, the very population with complex healthcare needs. As is stated, the widely adopted exclusion criterion may further limit the generalizability and slow the accrual in view of the significant barriers of participation in clinical trials $[4,5,9]$. And the plea to liberalize the exclusion criteria about a history of previous malignancy has been made by several working groups $[8,10,23]$. Accordingly, the current study was performed to assess the feasibility of enrolling patients with prior malignancy into clinical trials.

Arguments against the enrollment of patients with previous malignancy mainly centered on the concern that the inherently vulnerable patients may present worse survival outcomes and more serious adverse events and thus, jeopardize the development of a new drug. Consequently, under- 

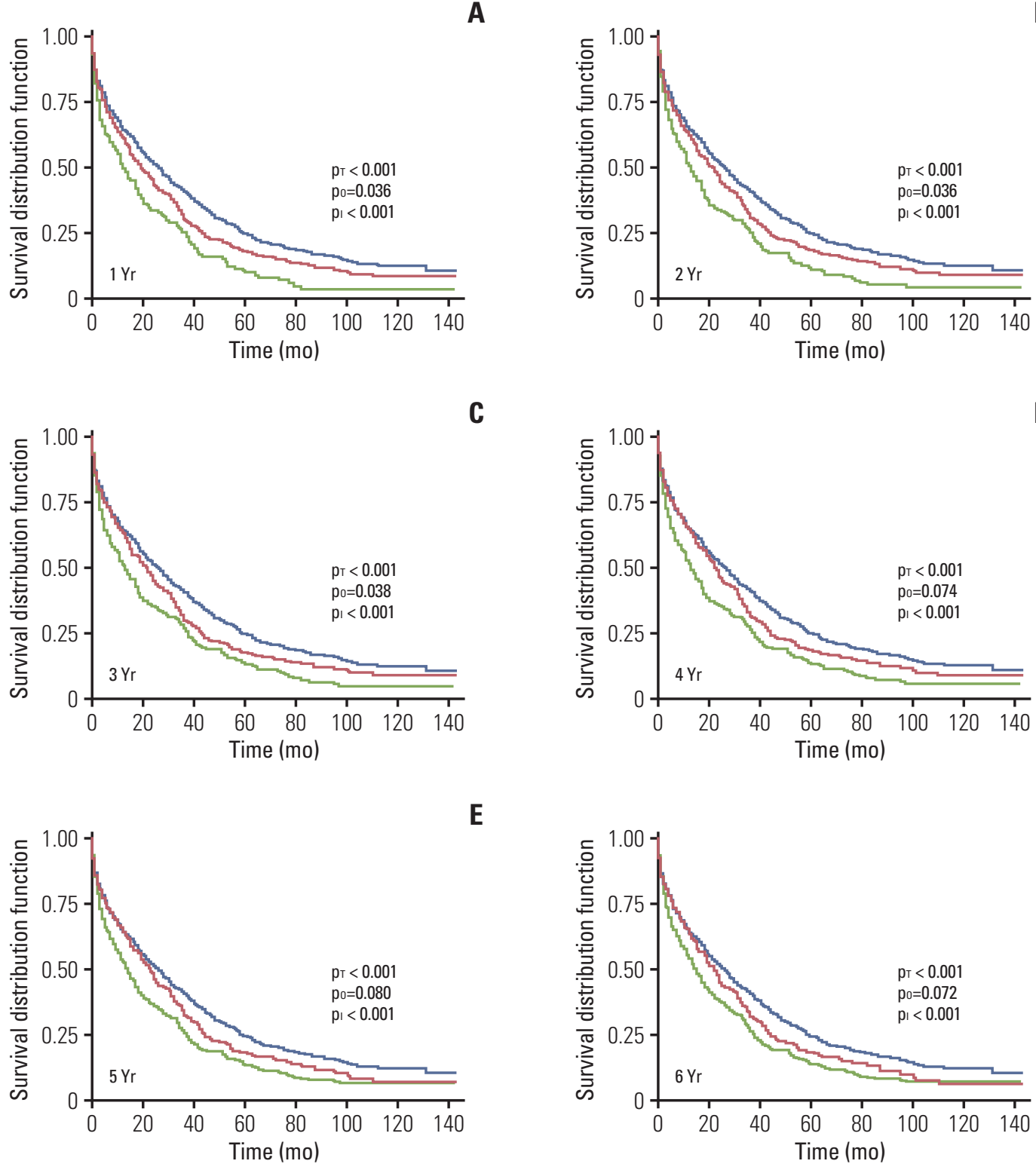

E

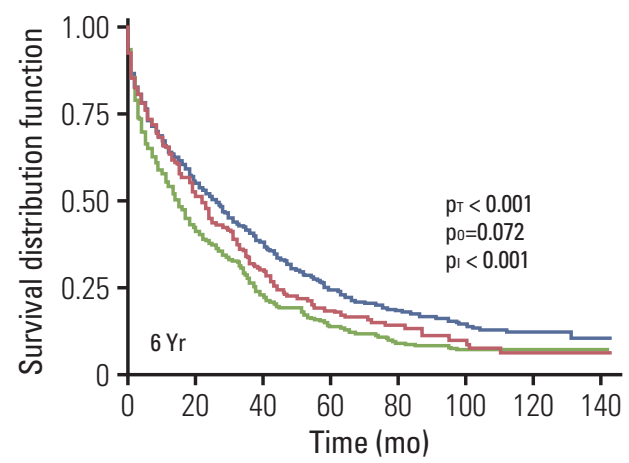

Fig. 3. Comparison of overall survival between patients with and without prior cancer. (A-J) The comparisons with different referenced timeframe ( 1 to 10 years) being adopted. The blue curves represent the patients without prior cancer; the red ones represent the patients with prior cancer diagnosed outside the referenced timeframe; the green ones represent the patients with prior cancer diagnosed inside the referenced timeframe. $\mathrm{p}_{\mathrm{T}}$ ( $\mathrm{p}_{\text {Total }}$ ) denotes the log-rank test for the total three curves; po (poutside) denotes the log-rank test between the red and blue curves; $p_{\text {I }}$ ( pInside $_{\text {) }}$ denotes the log-rank test between the green and blue curves. (Continued to the next page)

standing the impact of prior malignancy is of clinical importance. Several studies have reported detailed results for specific sites of the index cancer, including lung cancer, prostate cancer and gastrointestinal cancer $[9,11,12,24,25]$. Still, there is no study demonstrating the impact of the prior cancer for patients with $A B C$. In this retrospective study, we found that a history of prior cancer might impair the survival of women with advanced disease in the overall population. After adjustment for propensity scores, these patients had approximately a quarter increased risks of mortality. On the contrary, a pan-cancer study by Zhou et al. [5] reported that pati-ents with stage IV breast cancer who carried a history of 
G

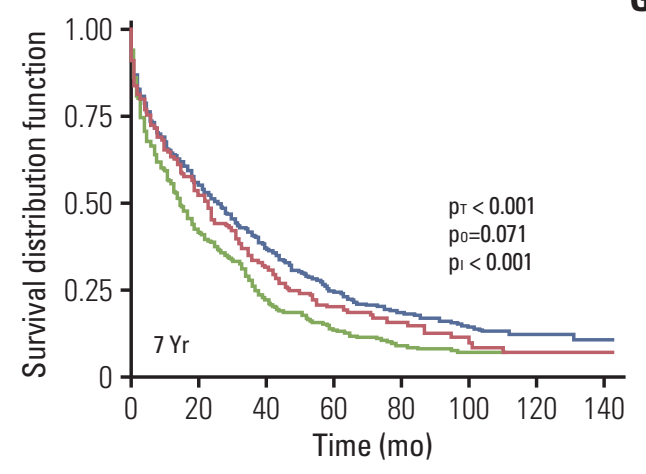

I

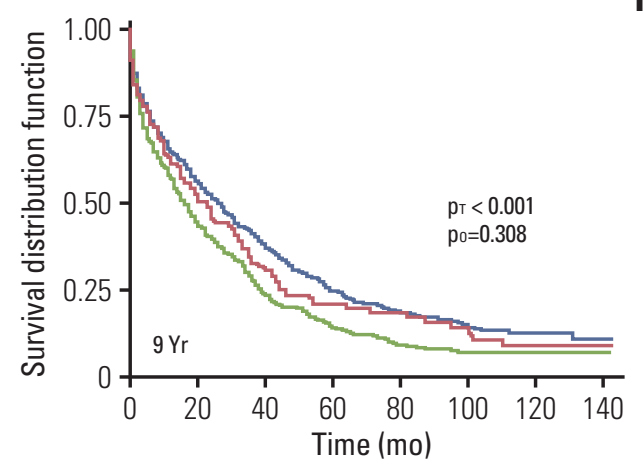

Fig. 3. (Continued from the previous page)

previous malignancy showed similar OS to those without a prior cancer $(\mathrm{p}=0.48)$. The discrepancy could be attributed to the different selection criteria. Our study excluded patients with a prior breast cancer diagnosis, which usually presented a more favorable prognosis when compared to other prior cancer types [1].

In the subgroup analysis, a previous malignancy at in situ or localized stage did not affect the survival adversely. In the current study, prior malignancy diagnosed at in situ or localized stage made up $53.6 \%$ of the overall population, suggesting that a substantial proportion of patients with advanced disease who carried a prior cancer history might be considered for enrollment into clinical trials. A possible explanation might be that the early-stage prior cancer presented less invasive tumor biology when compared to the advanced breast cancer and thus, less prior cancer-related mortality would be observed. Consistently, a study by Schonberg et al. [26] showed that women age 67 years or older diagnosed with ductal carcinoma in situ or stage I breast cancer presented a slightly better survival than their matched controls without a diagnosis of breast cancer. As is reported, higher 5-year relative survival rates are observed for localized genital, digestive, respiratory or urinary cancer when compared to $A B C$ [1]. Hence, it may be speculated that patients with

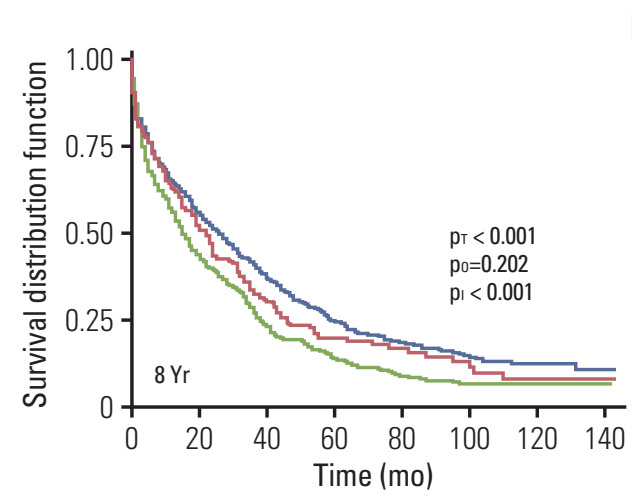

H

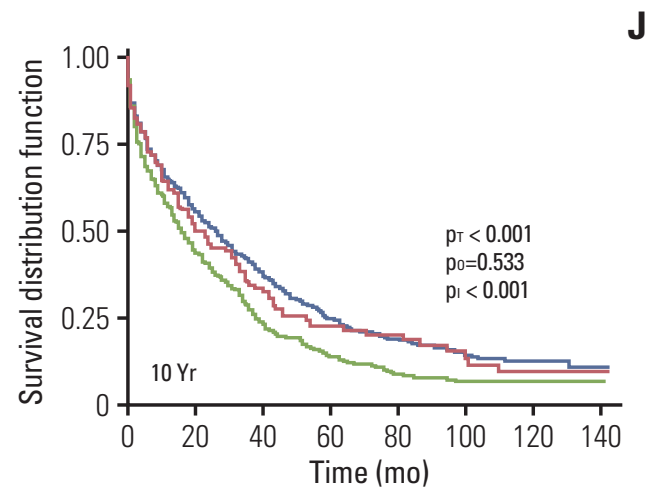

J

aggressive disease would not suffer significant detriment to survival from an earlier-stage prior cancer with low invasiveness. Furthermore, cancers at early stage usually response better to local and systemic treatment [11]. Given the early diagnosis, indolent nature, and the great treatment response, it seems reasonable that patients with early-stage prior cancers may present a favorable prognosis.

The time elapsed between the diagnosis of prior cancer and index breast cancer are a factor of great importance when assessing the effect of prior cancer [5]. In order to find the optimal time window, we performed survival analysis comparing OS between patients without a prior cancer history and those with prior cancer diagnosed outside the referenced timeframe. Finally, descending HRs were observed with the ascending diagnosis interval and we found that patients with a previous malignancy diagnosed four years or longer before the breast cancer showed similar survival to those without previous malignancy diagnosis. As is reported, notable decline is observed in deaths or relapse occurred in years 5-10 when compared to years 1-5 among the aggressive epithelial cancers such as ovarian cancer, gastric cancer and lung cancer [27-29]. Consequently, we presume it that there is a time point beyond which the prior cancer may be considered cured to some extent if no relapse or progression 
Table 2. Association of timing, stage, and type of prior cancer with overall and breast cancer-specific survival

\begin{tabular}{|c|c|c|c|c|c|c|}
\hline \multirow{2}{*}{ Variable } & \multicolumn{3}{|c|}{ Overall survival } & \multicolumn{3}{|c|}{ Breast cancer-specific survival } \\
\hline & Events/Cases & HR $(95 \%$ CI $)$ & p-value & Events/Cases & SHR $(95 \% \mathrm{CI})$ & p-value \\
\hline \multicolumn{7}{|c|}{$\begin{array}{l}\text { Part I: Prior cancer diagnosed outside } \\
\text { the referenced time frame } \\
\text { (vs. no prior cancer) }\end{array}$} \\
\hline $1 \mathrm{Yr}$ & $124 / 132$ & $1.21(1.04-1.40)$ & 0.015 & $59 / 132$ & $0.93(0.79-1.10)$ & 0.396 \\
\hline $2 \mathrm{Yr}$ & $162 / 173$ & $1.18(1.01-1.38)$ & 0.036 & $84 / 173$ & $0.94(0.79-1.12)$ & 0.512 \\
\hline $3 \mathrm{Yr}$ & $190 / 205$ & $1.19(1.01-1.39)$ & 0.038 & $104 / 205$ & $0.96(0.80-1.16)$ & 0.694 \\
\hline $4 \mathrm{Yr}$ & $213 / 231$ & $1.16(0.98-1.37)$ & 0.074 & $119 / 231$ & $0.98(0.81-1.18)$ & 0.836 \\
\hline $5 \mathrm{Yr}$ & $243 / 266$ & $1.16(0.98-1.38)$ & 0.080 & $140 / 266$ & $1.01(0.84-1.23)$ & 0.887 \\
\hline $6 \mathrm{Yr}$ & $268 / 295$ & $1.18(0.99-1.41)$ & 0.072 & $156 / 295$ & $1.09(0.89-1.33)$ & 0.427 \\
\hline $7 \mathrm{Yr}$ & $289 / 317$ & $1.14(0.94-1.38)$ & 0.171 & $170 / 317$ & $1.13(0.92-1.39)$ & 0.275 \\
\hline $8 \mathrm{Yr}$ & $302 / 330$ & $1.14(0.93-1.40)$ & 0.202 & $181 / 330$ & $1.11(0.89-1.39)$ & 0.347 \\
\hline $9 \mathrm{Yr}$ & $324 / 355$ & $1.12(0.90-1.40)$ & 0.308 & $201 / 355$ & $1.06(0.83-1.36)$ & 0.639 \\
\hline $10 \mathrm{Yr}$ & $333 / 364$ & $1.08(0.85-1.36)$ & 0.533 & $210 / 364$ & $0.99(0.76-1.28)$ & 0.928 \\
\hline \multicolumn{7}{|c|}{$\begin{array}{l}\text { Part II: Prior cancer stage } \\
\text { (vs. no prior cancer) }\end{array}$} \\
\hline In situ & $7 / 9$ & $1.02(0.48-2.18)$ & 0.962 & $7 / 9$ & $1.41(0.68-2.90)$ & 0.353 \\
\hline Localized & $215 / 241$ & $1.15(0.97-1.36)$ & 0.083 & $172 / 241$ & $1.06(0.89-1.27)$ & 0.516 \\
\hline Regional & $109 / 121$ & $1.32(1.07-1.64)$ & 0.010 & $60 / 121$ & $0.61(0.46-0.80)$ & $<0.001$ \\
\hline Distant & $70 / 73$ & $1.84(1.40-2.42)$ & $<0.001$ & $27 / 73$ & $0.40(0.27-0.60)$ & $<0.001$ \\
\hline Unstaged & $19 / 22$ & $1.34(0.85-2.13)$ & 0.210 & $13 / 22$ & $0.86(0.49-1.50)$ & 0.587 \\
\hline \multicolumn{7}{|c|}{$\begin{array}{l}\text { Part III: Prior cancer type } \\
\text { (vs. no prior cancer) }\end{array}$} \\
\hline Digestive & 99/109 & $1.29(1.03-1.61)$ & 0.025 & $61 / 109$ & $0.71(0.54-0.93)$ & 0.013 \\
\hline Head and neck & $7 / 8$ & $1.24(0.54-2.83)$ & 0.618 & $4 / 8$ & $0.63(0.22-1.77)$ & 0.379 \\
\hline Respiratory & $53 / 56$ & $2.08(1.50-2.87)$ & $<0.001$ & $25 / 56$ & $0.58(0.37-0.91)$ & 0.017 \\
\hline Genital & $140 / 151$ & $1.29(1.07-1.55)$ & 0.007 & $97 / 151$ & $0.86(0.69-1.08)$ & 0.194 \\
\hline Urinary & $48 / 52$ & $1.64(1.22-2.21)$ & 0.001 & $29 / 52$ & $0.75(0.51-1.11)$ & 0.151 \\
\hline Endocrine & $23 / 31$ & $0.89(0.56-1.42)$ & 0.630 & $21 / 31$ & $1.05(0.67-1.66)$ & 0.819 \\
\hline Other & $50 / 59$ & $0.99(0.76-1.29)$ & 0.958 & $42 / 59$ & $1.00(0.75-1.34)$ & 0.996 \\
\hline
\end{tabular}

$\mathrm{HR}$, hazard ratio; $\mathrm{CI}$, confidence interval; $\mathrm{SHR}$, subdistribution hazard ratio.

observed, accounting for no survival detriment from prior cancer diagnosed outside the timeframe of four years. In our study, more than half of the prior malignancy occurred four years or longer before the breast cancer diagnosis, indicating that a specific subgroup of patients with prior cancer history was eligible for breast cancer trials.

Also, our study revealed that type of previous malignancy may affect both OS and BCSS significantly. From our perspective, the OS of patients with prior cancer is determined by the cancer with more aggressive biology between prior cancer and index cancer. As is reported by Zhou et al. [5], cancers with relatively good prognoses are more inclined to be affected by prior malignancy, whereas the outcomes of aggressive cancers are usually completely unrelated to prior cancer. Our work revealed that prior cancers of digestive, respiratory, genital, and urinary system had an adverse effect on the prognosis for $\mathrm{ABC}$ patients. According to Cancer Statistics 2018, breast cancer presents more favorable prognosis than these cancers listed above [1]. This may be the reason why different cancer types have different effects on OS.

As is expected, a previous malignancy diagnosis was associated with significantly higher prior cancer-related mortality, leading to the worse OS of those patients. However, better BCSS in the overall population was observed for those with prior malignancy for some uncovered reasons when using the propensity scores-adjusted competing risks regression. Analogous results were presented in each subgroup. One possible explanation is that prior cancer-specific mortality is a competing event to breast cancer-specific mortality. According to Cancer Statistics 2018, female breast cancer is one of the cancers with the highest survival when comparing with cancers from female genitourinary, gastrointestinal, or 
Table 3. Propensity score-adjusted multivariable analysis of overall and breast cancer-specific survival among patients with prior cancer

\begin{tabular}{|c|c|c|c|c|}
\hline & HR $(95 \%$ CI) & p-value & $\begin{array}{c}\text { Breast cancer-specific } \\
\text { SHR }(95 \% \text { CI })\end{array}$ & $\begin{array}{c}\text { Breast cancer-specific } \\
\text { p-value }\end{array}$ \\
\hline \multicolumn{5}{|l|}{ Prior cancer history } \\
\hline No & Reference & - & Reference & - \\
\hline Yes & $1.23(1.07-1.41)$ & 0.004 & $0.75(0.63-0.88)$ & $<0.001$ \\
\hline \multicolumn{5}{|l|}{ Age (yr) } \\
\hline$<40$ & Reference & - & - & - \\
\hline $40-64$ & $1.14(0.53-2.45)$ & 0.737 & - & - \\
\hline$>64$ & $1.55(0.72-3.35)$ & 0.262 & - & - \\
\hline \multicolumn{5}{|l|}{ Marital status } \\
\hline Married & Reference & - & Reference & - \\
\hline Single & $1.01(0.80-1.28)$ & 0.914 & $1.01(0.78-1.31)$ & 0.950 \\
\hline Sep/wid/div & $1.24(1.06-1.45)$ & 0.009 & $1.22(1.02-1.47)$ & 0.031 \\
\hline Unknown & $0.96(0.67-1.36)$ & 0.815 & $0.87(0.56-1.36)$ & 0.544 \\
\hline \multicolumn{5}{|l|}{ Grade } \\
\hline G1 & Reference & - & Reference & - \\
\hline G2 & $1.37(1.01-1.87)$ & 0.046 & $1.42(1.00-2.00)$ & 0.048 \\
\hline G3/G4 & $1.62(1.19-2.21)$ & 0.002 & $1.53(1.09-2.17)$ & 0.015 \\
\hline Unknown & $1.33(0.95-1.87)$ & 0.092 & $1.13(0.77-1.66)$ & 0.539 \\
\hline \multicolumn{5}{|l|}{ ER } \\
\hline Positive & Reference & - & - & - \\
\hline Negative & $1.40(1.11-1.75)$ & 0.004 & - & - \\
\hline Unknown & $1.64(0.81-3.30)$ & 0.167 & - & - \\
\hline \multicolumn{5}{|l|}{ PR } \\
\hline Positive & Reference & - & Reference & - \\
\hline Negative & $1.39(1.13-1.70)$ & 0.002 & $1.20(1.00-1.44)$ & 0.054 \\
\hline Unknown & $1.16(0.59-2.27)$ & 0.670 & $1.30(0.98-1.44)$ & 0.066 \\
\hline \multicolumn{5}{|l|}{ Treatment } \\
\hline No & Reference & - & Reference & - \\
\hline Surg only & $0.63(0.44-0.65)$ & $<0.001$ & $0.54(0.42-0.69)$ & $<0.001$ \\
\hline Chemo only & $0.68(0.56-0.83)$ & $<0.001$ & $0.95(0.76-1.18)$ & 0.614 \\
\hline Surg and chemo & $0.31(0.25-0.39)$ & $<0.001$ & $0.58(0.46-0.73)$ & $<0.001$ \\
\hline
\end{tabular}

HR, hazard ratio; CI, confidence interval; SHR, subdistribution hazard ratio; Sep/wid/div, separated/widowed/divorced; ER, estrogen receptor; PR, progesterone receptor; Surg, surgery; Chemo, chemotherapy.

respiratory systems, which account for the majority of the prior cancers in our study [1]. Accordingly, the growing in prior cancer-related mortality (events of competing risks) leads to the decline in breast cancer-specific mortality (events of interest). Another potential explanation is the lead-time bias [11]. Patients diagnosed with prior cancer might seek routine medical attention to the prior cancer, allowing for early diagnosis in the disease course [26]. This explanation echoed the previous study by Ahn et al. [30] that increased comorbidity burden is associated with earlier cancer diagnosis. In terms of treatment, some regimens for systemic therapy used in gastrointestinal or respiratory cancers may also work in ABC. In spite of these speculations, the relatively bet- ter prognosis of breast cancer along with the competing relationship between breast cancer-specific mortality and prior cancer-related mortality may be the main reasons and work together leading to the lower OS and higher BCSS for patients with previous malignancy. Still, further investigation is required.

Another reason for excluding prior cancer from breast cancer trials is that preexposure to the prior cancer treatment may interfere with the trial outcomes, such as the efficacy and adverse events profiles. However, it is hard to address the issue in our study due to the limited data in the SEER database.

Still, there are several limitations of our work. First, some 
variables were not available in the SEER database, such as the comorbidities. So it is difficult to match the comorbidities and other unobserved confounding factors in the PSM analyses. Any hidden bias owing to latent variables remained after matching. Neither did we include these variables into the multivariable regression model. Second, SEER did not provide detailed information on treatment, making the adjustment impossible for both locoregional and systemic treatment. In addition, after excluding patients with a prior breast cancer diagnosis, a small sample size of patients with previous malignancy were identified and thereby, limited the generalizability to those with second primary breast cancer. Further investigation is required. Furthermore, as is stated above, we could not address the issue of the efficacy and adverse events profiles of prior cancer treatment.

In conclusion, a substantial proportion of patients with stage IV breast cancer have a history of prior malignancy. More than half of these cases are diagnosed at in situ or localized stage outside the timeframe of 4 years. These patients may be considered for enrollment in clinical trials of $\mathrm{ABC}$ since no survival detriment was observed. It also suggests a broader inclusion criterion and emphasizes the importance that exclusion criteria should be modified based on specific stage, type and timing of prior cancer rather than being duplicated from prior trials. Only in this way can patients with a history of prior cancer get access to the stateof-the-art therapies.

\section{Electronic Supplementary Material}

Supplementary materials are available at Cancer Research and Treatment website (https: // www.e-crt.org).

\section{Conflicts of Interest}

Conflict of interest relevant to this article was not reported.

\section{Acknowledgments}

The study was supported by National Natural Science Foundation of China (No. 81572581) and Science and Technology Commission of Shanghai Municipality Project (No. 16411966900). The funding source had no role in the design of this study and will not have any role during its execution, analyses, interpretation of the data, writing assistance, or decision to submit results.

\section{References}

1. Siegel RL, Miller KD, Jemal A. Cancer statistics, 2018. CA Cancer J Clin. 2018;68:7-30.

2. Jemal A, Ward EM, Johnson CJ, Cronin KA, Ma J, Ryerson B, et al. Annual report to the nation on the status of cancer, 19752014, featuring survival. J Natl Cancer Inst. 2017;109:djx030.

3. Hayat MJ, Howlader N, Reichman ME, Edwards BK. Cancer statistics, trends, and multiple primary cancer analyses from the Surveillance, Epidemiology, and End Results (SEER) Program. Oncologist. 2007;12:20-37.

4. Murphy CC, Gerber DE, Pruitt SL. Prevalence of prior cancer among persons newly diagnosed with cancer: an initial report from the Surveillance, Epidemiology, and End Results program. JAMA Oncol. 2018;4:832-6.

5. Zhou H, Huang Y, Qiu Z, Zhao H, Fang W, Yang Y, et al. Impact of prior cancer history on the overall survival of patients newly diagnosed with cancer: a pan-cancer analysis of the SEER database. Int J Cancer. 2018;143:1569-77.

6. National Comprehensive Cancer Network (NCCN). Clinical Practice Guidelines in Oncology: Breast Cancer. version 3.2018 [Internet]. Plymouth Meeting, PA: National Comprehensive Cancer Network; 2018 [cited 2018 Nov 20]. Available from: http: // NCCN.org.

7. Lemieux J, Goodwin PJ, Pritchard KI, Gelmon KA, Bordeleau LJ, Duchesne T, et al. Identification of cancer care and protocol characteristics associated with recruitment in breast cancer clinical trials. J Clin Oncol. 2008;26:4458-65.

8. Kim ES, Bruinooge SS, Roberts S, Ison G, Lin NU, Gore L, et al. Broadening eligibility criteria to make clinical trials more representative: American Society of Clinical Oncology and Friends of Cancer Research Joint Research Statement. J Clin Oncol. 2017;35:3737-44.

9. Gerber DE, Laccetti AL, Xuan L, Halm EA, Pruitt SL. Impact of prior cancer on eligibility for lung cancer clinical trials. J Natl Cancer Inst. 2014;106:dju302.

10. The Food and Drug Administration. Cancer Clinical Trial Eligibility Criteria: Patients with Organ Dysfunction or Prior or Concurrent Malignancies [Internet]. Washington, DC: The Food and Drug Administration; c2019 [cited 2019 Mar 7]. Available from: https://www.fda.gov/Drugs/GuidanceComplianceRegulatoryInformation/Guidances/default.htm.

11. Laccetti AL, Pruitt SL, Xuan L, Halm EA, Gerber DE. Effect of prior cancer on outcomes in advanced lung cancer: implications for clinical trial eligibility and accrual. J Natl Cancer Inst. 2015;107:djv002.

12. Laccetti AL, Pruitt SL, Xuan L, Halm EA, Gerber DE. Prior cancer does not adversely affect survival in locally advanced lung cancer: a national SEER-medicare analysis. Lung Cancer. 2016; 98:106-13.

13. Gerber DE, Pruitt SL, Halm EA. Should criteria for inclusion in cancer clinical trials be expanded? J Comp Eff Res. 2015;4: 
289-91.

14. Shameem R, Hamid MS, Mavani GP, Singhal N, Shani D, Sullivan KM. Secondary primary cancers in male breast cancer survivors. J Clin Oncol. 2014;32(26 Suppl):129.

15. Gray RJ. A class of K-sample tests for comparing the cumulative incidence of a competing risk. Ann Stat. 1988;16:1141-54.

16. Fine JP, Gray RJ. A proportional hazards model for the subdistribution of a competing risk. J Am Stat Assoc. 1999;94:496509.

17. Scrucca L, Santucci A, Aversa F. Regression modeling of competing risk using R: an in depth guide for clinicians. Bone Marrow Transplant. 2010;45:1388-95.

18. Schoenfeld D. Partial residuals for the proportional hazards regression model. Biometrika. 1982;69:239-41.

19. Austin PC. An introduction to propensity score methods for reducing the effects of confounding in observational studies. Multivariate Behav Res. 2011;46:399-424.

20. Yang D, Dalton JE. A unified approach to measuring the effect size between two groups using SAS. Orlando, FL: SAS Global Forum; 2012. p. 1-6.

21. Davidson NE. Incident cancer in cancer survivors: when cancer lurks in the background. JAMA Oncol. 2018;4:836-7.

22. Bluethmann SM, Mariotto AB, Rowland JH. Anticipating the "Silver Tsunami": prevalence trajectories and comorbidity burden among older cancer survivors in the United States. Cancer Epidemiol Biomarkers Prev. 2016;25:1029-36.

23. Ledford H. Cancer researchers push to relax rules for clinical trials. Nature. 2018;556:12-3.
24. Dinh KT, Mahal BA, Ziehr DR, Muralidhar V, Chen YW, Viswanathan $\mathrm{VB}$, et al. Risk of prostate cancer mortality in men with a history of prior cancer. BJU Int. 2016;117:E20-8.

25. Smyth EC, Tarazona N, Peckitt C, Armstrong E, Mansukhani S, Cunningham D, et al. Exclusion of gastrointestinal cancer patients with prior cancer from clinical trials: is this justified? Clin Colorectal Cancer. 2016;15:e53-9.

26. Schonberg MA, Marcantonio ER, Ngo L, Li D, Silliman RA, McCarthy EP. Causes of death and relative survival of older women after a breast cancer diagnosis. J Clin Oncol. 2011;29: 1570-7.

27. Baldwin LA, Huang B, Miller RW, Tucker T, Goodrich ST, Podzielinski I, et al. Ten-year relative survival for epithelial ovarian cancer. Obstet Gynecol. 2012;120:612-8.

28. Lee JH, Kim HI, Kim MG, Ha TK, Jung MS, Kwon SJ. Recurrence of gastric cancer in patients who are disease-free for more than 5 years after primary resection. Surgery. 2016;159: 1090-8.

29. Shintani T, Matsuo Y, Iizuka Y, Mitsuyoshi T, Mizowaki T. A retrospective long-term follow-up study of stereotactic body radiation therapy for non-small cell lung cancer from a single institution: incidence of late local recurrence. Int J Radiat Oncol Biol Phys. 2018;100:1228-36.

30. Ahn DH, Mehta N, Yorio JT, Xie Y, Yan J, Gerber DE. Influence of medical comorbidities on the presentation and outcomes of stage I-III non-small-cell lung cancer. Clin Lung Cancer. 2013;14:644-50. 